\title{
Guiding Dynamic Symbolic Execution toward Unverified Program Executions
}

\author{
Maria Christakis $\quad$ Peter Müller $\quad$ Valentin Wüstholz \\ Department of Computer Science, ETH Zurich, Switzerland \\ \{maria.christakis, peter.mueller, valentin.wuestholz\}@inf.ethz.ch
}

\begin{abstract}
Most techniques to detect program errors, such as testing, code reviews, and static program analysis, do not fully verify all possible executions of a program. They leave executions unverified when they do not check certain properties, fail to verify properties, or check properties under certain unsound assumptions such as the absence of arithmetic overflow.

In this paper, we present a technique to complement partial verification results by automatic test case generation. In contrast to existing work, our technique supports the common case that the verification results are based on unsound assumptions. We annotate programs to reflect which executions have been verified, and under which assumptions. These annotations are then used to guide dynamic symbolic execution toward unverified program executions. Our main technical contribution is a code instrumentation that causes dynamic symbolic execution to abort tests that lead to verified executions, to prune parts of the search space, and to prioritize tests that cover more properties that are not fully verified. We have implemented our technique for the .NET static analyzer Clousot and the dynamic symbolic execution tool Pex. It produces smaller test suites (by up to 19.2\%), covers more unverified executions (by up to 7.1\%), and reduces testing time (by up to $52.4 \%$ ) compared to combining Clousot and Pex without our technique.
\end{abstract}

\section{INTRODUCTION}

Modern software projects use a variety of techniques to detect program errors, such as testing, code reviews, and static program analysis [31]. In practice, none of these techniques check all possible executions of a program. They often leave entire paths unverified (for instance, when a test suite does not achieve full path coverage), fail to verify certain properties (such as complex assertions), or verify some paths under assumptions (such as the absence of arithmetic overflow) that might not hold on all executions of the path. Making such assumptions is necessary in code reviews to reduce the complexity of the task; it is also customary in static

Permission to make digital or hard copies of all or part of this work for personal or classroom use is granted without fee provided that copies are not made or distributed for profit or commercial advantage and that copies bear this notice and the full citation on the first page. Copyrights for components of this work owned by others than the author(s) must be honored. Abstracting with credit is permitted. To copy otherwise, or republish, to post on servers or to redistribute to lists, requires prior specific permission and/or a fee. Request permissions from permissions@ acm.org.

ICSE '16, May 14 - 22, 2016, Austin, TX, USA

(C) 2016 Copyright held by the owner/author(s). Publication rights licensed to ACM. ISBN 978-1-4503-3900-1/16/05 . .\$15.00

DOI: http://dx.doi.org/10.1145/2884781.2884843

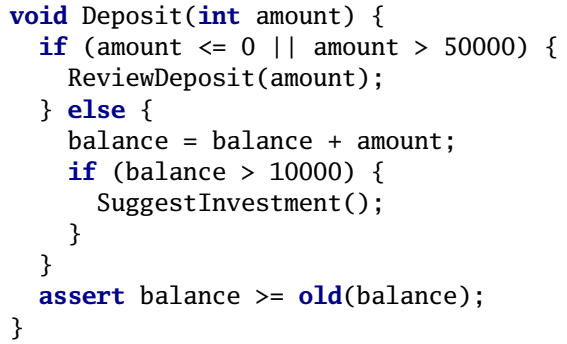

Figure 1: C\# example illustrating partial verification results. Techniques that assume that the addition on line 5 does not overflow might miss violations of the assertion on line 10 . We use the assertion to make the intended behavior explicit; the old keyword indicates that an expression is evaluated in the pre-state of the method. balance is an integer field declared in the enclosing class. We assume methods ReviewDeposit and SuggestInvestment to be correct.

program analysis to improve the precision, performance, and modularity of the analysis [13], and because some program features elude static checking [36]. That is, most static analyses sacrifice soundness in favor of other important qualities.

Automatic test case generation via dynamic symbolic execution (DSE) [27, 9], also called concolic testing [38], systematically explores a large number of program executions and, thus, effectively detects errors missed by other techniques. However, simply applying DSE in addition to other techniques leads to redundancy when executions covered by DSE have already been verified. In this case, the available testing time is wasted on executions that are known to be correct rather than on exploring previously-unverified executions. This redundancy is especially problematic when DSE is used to complement static analyzers because static techniques can check a large fraction of all possible program executions and, thus, many or even most of the executions covered by DSE are already verified.

Method Deposit in Fig. 1 illustrates this problem. A reviewer or static analyzer that checks the implementation under the assumption that the addition on line 5 does not overflow might miss violations of the assertion on line 10 . Applying DSE to the method tries to explore six different paths through the method (there are three paths through the conditionals, each combined with two possible outcomes for the assertion), in addition to all the paths through the called methods ReviewDeposit and SuggestInvestment. Assuming that these two methods are correct, only one of all these paths reveals an error, namely the path that is taken when amount is between 0 and 50,000, and balance is large 
enough for the addition on line 5 to overflow. All other generated test cases are redundant because they lead to executions that have already been verified. In particular, if the called methods have complex control flow, DSE might not detect the error because it reaches a timeout before generating the only relevant test case.

To reduce this redundancy, existing work [10, 17, 25] integrates static analyses and DSE; it uses the verification results of a static analysis to prune verified executions from testing. However, existing combinations of static analysis and test case generation do not support analyses that make unsound assumptions. They either require the static analysis to be sound and are thus of limited use for most practical analyses, or they ignore the unsoundness of the static analysis and may therefore prune executions during DSE that contain errors. In particular, they would miss the error in Fig. 1 if the static analysis ignores overflow.

In this paper, we present a novel technique to complement partial verification by automatic test case generation. In contrast to existing work, our technique supports the important case that the verification results are obtained by an unsound (manual or automatic) static analysis. Building on our earlier work [12], we use program annotations to make explicit which assertions in a program have already been verified, and under which assumptions. These annotations can be generated automatically by a static analysis [13] or inserted manually, for instance, during a code review. We consider a code reviewer as a human static analyzer, since like tools, reviewers typically make simplifying assumptions. The main technical contribution of this paper is a code instrumentation of the unit under test that (1) detects redundant test cases early during their execution and aborts them, (2) reduces the search space for DSE by pruning paths that have been previously verified, and (3) prioritizes test cases that cover more assertions that are not fully verified. This instrumentation is based on an efficient static inference that propagates information about unverified executions up in the control flow, where it may prune the search space more effectively. It does not require a specific DSE algorithm and, thus, can be used with a wide range of existing tools.

This paper goes beyond our previous work [12] in three important ways: (1) It leverages partial verification results, whereas our previous work reduced the test effort mainly for fully-verified methods. Practical analyses typically do not achieve full verification for non-trivial methods. (2) It demonstrates the effectiveness of our approach using an industrial analyzer and the sources of unsoundness it contains; our previous work used an artificially-unsound variation of Dafny [34]. (3) It provides a more substantial evaluation.

Our technique works for modular and whole-program verification, and can be used to generate unit or system tests. We present it for modular verification and unit testing here. In particular, we have implemented our approach for Microsoft's .NET static checker Clousot [23], a modular static analysis, and the DSE tool Pex [40], a test case generator for unit tests. Our experiments demonstrate that, compared to classical DSE, our approach produces smaller test suites, explores more unverified executions, and reduces testing time.

Outline. We give an overview of our approach in Sect. 2 . Sect. 3 explains how we infer the code instrumentation from partial verification results. Our experimental results are presented in Sect. 4. We discuss related work in Sect. 5 and conclude in Sect. 6 .

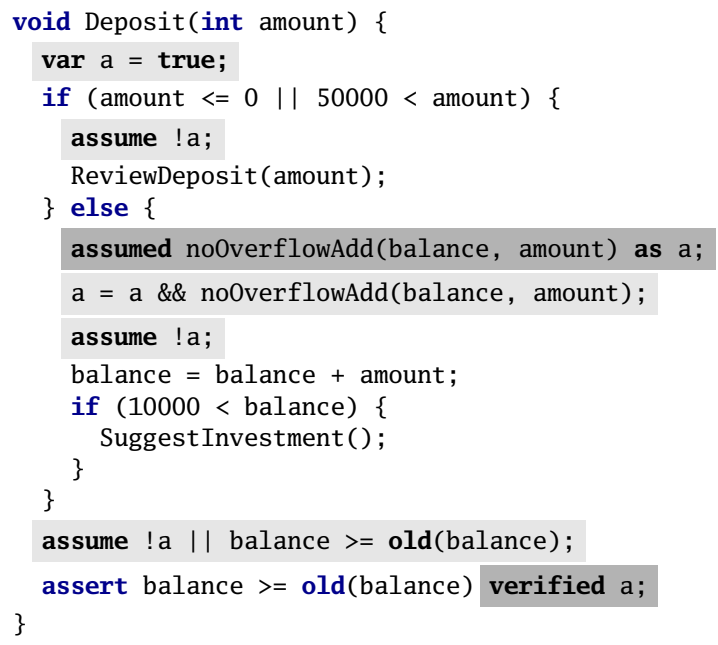

Figure 2: The instrumented version of the method from Fig. 1. The dark boxes show the annotations generated by the static analyzer. The assumed statement makes explicit that the analyzer assumed that the addition on line 10 does not overflow. The verified annotation on the assertion on line 16 expresses that the assertion was verified under this unsound assumption. The two annotations are connected via the assumption identifier a, which uniquely identifies the assumed statement. The light boxes show the instrumentation that we infer from the annotations and that prunes redundant tests.

\section{APPROACH}

In this section, we summarize an annotation language that we have developed in earlier work $[12,41]$ to express partial verification results, and then illustrate how the instrumentation proposed here uses these annotations to guide DSE toward unverified executions. The details of the approach are explained in the next section.

\subsection{Verification annotations}

In order to encode partial verification results, we introduce two kinds of annotations: An assumed statement of the form assumed $P$ as $a$ expresses that an analysis assumed property $P$ to hold at this point in the code without checking it. The assumption identifier a uniquely identifies this statement. In order to record verification results, we use assertions of the form assert $P$ verified $A$, which express that property $P$ has been verified under condition $A$. The premise $A$ is a boolean condition over assumption identifiers, each of which is introduced in an assumed statement. Specifically, it is the conjunction of the identifiers for the assumptions used to verify $P$, or false if $P$ was not verified. When several verification results are combined (for instance, from a static analysis and a code review), $A$ is the disjunction of the assumptions made during each individual verification. We record verification results for all assertions in the code, including implicit assertions such as a receiver being non-null or an index being within the bounds of an array.

We assume here that a static analyzer records the assumptions it made during the analysis, which assertions it verified, and under which assumptions. We equipped Microsoft's .NET static analyzer Clousot [23] with this functionality [13]. Among other unsound assumptions, Clousot ignores arithmetic overflow and, thus, misses the potential 
violation of the assertion on line 10 of Fig. $1^{1}$. This partial verification result is expressed by the annotations in the dark boxes of Fig. 2 (the light boxes are discussed below). The assumed statement makes explicit that the addition on line 10 was assumed not to overflow (the predicate no0verflowAdd can be encoded as equality of an integer and a long-integer addition); the verified annotation on the assertion on line 16 expresses that the assertion was verified under this (unsound) assumption.

The meaning of verification annotations is defined in terms of assignments and standard assume statements, which makes the annotations easy to support by a wide range of static and dynamic tools. For each assumption identifier, we declare a boolean variable, which is initialized to true. For modular analyses, assumption identifiers are local variables initialized at the beginning of the enclosing method (line 2 in Fig. 2); for whole-program analyses, assumption identifiers are global variables initialized for instance during class initialization. A statement assumed $P$ as $a$ is encoded as

$$
a=a \& \& P
$$

as illustrated on line 8 . That is, variable $a$ accumulates the assumed properties for each execution of the assumed statement. Since assumptions typically depend on the current execution state, this encoding ensures that an assumption is evaluated in the state in which it is made rather than the state in which it is used.

An assertion assert $P$ verified $A$ is encoded as

$$
\begin{aligned}
& \text { assume } A \Rightarrow P ; \\
& \text { assert } P ;
\end{aligned}
$$

as illustrated on line 15 . The assume statement expresses that, if condition $A$ holds, then the asserted property $P$ holds as well, which reflects that $P$ was verified under the premise $A$. Consequently, an assertion is unverified if $A$ is false, the assertion is fully verified if $A$ is true, and otherwise, the assertion is partially verified.

\subsection{Guiding dynamic symbolic execution}

To reduce redundancies with prior analyses of the unit under test, DSE should generate test cases that check each assertion assert $P$ verified $A$ for the case that the premise $A$ does not hold, because $P$ has been verified to hold otherwise. We guide DSE toward such test cases by pruning test cases that cover verified executions. Moreover, we prioritize test cases that violate more assertion premises and, thus, are more likely to reveal an assertion violation. Test prioritization is important when DSE is applied until certain limits (for instance, on the overall testing time) are reached.

Pruning redundant tests. A test is redundant if the premise of each assertion in its execution holds; in this case, all assertions have been statically verified. To prune redundant tests, we compute statically for each program point a sufficient condition for every execution from this program point onward to be verified. If this condition holds during the execution of a test case, all subsequent assertions are definitely verified and, thus, the test can be aborted. More importantly, all other test cases that share the prefix of the execution path up to the abort and also satisfy the condition

\footnotetext{
${ }^{1}$ Clousot is modular, that is, reasons about a method call using the method's pre- and postcondition; we assume here that the postconditions of ReviewDeposit and SuggestInvestment state that balance is not decreased.
}

can be pruned from the search space for DSE. If all assertions in the shared prefix are fully verified, then these test cases are redundant. Otherwise, they are not redundant according to the definition above, but nevertheless guaranteed not to reveal an assertion violation. Assertions after the shared prefix are definitely verified; violations of assertions in the shared prefix would be detected before aborting the former test case since DSE tools treat assertions as branches and, thus, two executions of the same path satisfy or violate the same assertions.

Both aborting and pruning of tests are achieved by instrumenting the unit under test with assume statements. They affect DSE in two ways. First, when the execution of a test case encounters an assume statement whose condition is false, the execution is aborted. Second, when an execution encounters an assume statement, its condition is added to the symbolic path condition, ensuring that subsequent test cases that share the prefix of the execution path up to the assume statement will satisfy the condition.

We instrument the unit under test by assuming at various program points a condition under which there may be an execution from this program point onward that is unverified. We call this condition a may-unverified condition; it is the negation of the condition that all executions from the point onward are verified. Note that this may-unverified instrumentation is conservative. It retains any execution in which the premise of at least one of the assertions might not hold. Therefore, it does not abort or prune any tests that may reveal an assertion violation.

The example in Fig. 2 has an assertion with premise a at the very end. Consider the program points on lines 4 and 9. At both points, a is a sufficient condition for the rest of the execution of Deposit to be verified. Since we are interested in test cases that lead to unverified executions, we instrument both program points by assuming the negation, !a. With this instrumentation, any test case that enters the outer then-branch is aborted since a is always true at this point, which, in particular, prunes the entire exploration of method ReviewDeposit. Similarly, any test case that does not lead to an overflow on line 10 is aborted on line 9, which prunes the entire exploration of method SuggestInvestment. So, out of all the test cases generated by DSE for the uninstrumented Deposit method, only the one that reveals the error remains; all others are either aborted early or pruned.

Note that the instrumentation aborts and prunes redundant tests more effectively if may-unverified conditions are assumed earlier in the control flow, because early assumptions may abort test cases earlier and share the prefix with more executions. For instance, if instead of the assumptions on lines 4 and 9 we assumed ! a only right before the assertion on line 16, tests would be aborted late and no redundant tests would be pruned. DSE would generate the same test cases as if there were no prior verification results. Our previous work [12] produces exactly this result, which demonstrates that it provides only weak support for partially-verified methods. To address this problem, we propagate constraints that characterize unverified executions higher up in the control flow, where they can be used to effectively prune redundant test cases.

Prioritizing premise violations. Intuitively, test cases that violate the premise of more than one assertion have a higher chance to detect an assertion violation. To prioritize such test cases, we devise a second instrumentation, called 


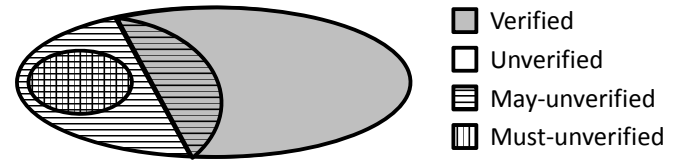

Figure 3: May-unverified and must-unverified conditions. The set of all executions is depicted by the large ellipse; the gray and white areas depict the verified and unverified executions, respectively. Executions that satisfy the may-unverified conditions are ruled horizontally, while those satisfying the mustunverified conditions are ruled vertically.

must-unverified instrumentation: We compute for each program point a sufficient condition for every execution from this program point onward to be definitely unverified. If the condition holds then every execution from the program point onward contains at least one assertion, and the premises of all assertions in the execution are false.

When the must-unverified condition is violated, it does not necessarily mean that the subsequent execution is verified and, thus, we cannot abort the test case. Therefore, we instrument the program not by assuming the must-unverified condition, but instead with a dedicated tryfirst statement. This statement interrupts the execution of the test case and instructs DSE to generate new inputs that satisfy the must-unverified condition, that is, inputs that have a higher chance to detect an assertion violation. The interrupted test case is re-generated later, after the executions that satisfy the must-unverified condition have been explored. This exploration strategy prioritizes test cases that violate all premises over those that violate only some.

Suppose that the Deposit method in Fig. 2 contained another assertion at the very end that has not been verified, that is, whose premise is false. In this case, the mayunverified instrumentation yields true for all prior program points since every execution is unverified. In this case, this instrumentation neither aborts nor prunes any test cases. In contrast, the must-unverified instrumentation infers !a on line 9. The corresponding tryfirst statement (not shown in Fig. 2) gives priority to executions that lead to an overflow on line 10. However, it does not prune the others since they might detect a violation of the unverified second assertion at the end of the method.

Summary. Fig. 3 illustrates the may-unverified and mustunverified conditions. The set of executions that satisfy the may-unverified conditions is a superset of the unverified executions, whereas the set of executions that satisfy the mustunverified conditions is a subset.

The may-unverified and must-unverified instrumentations have complementary strengths. While the former effectively aborts or prunes redundant tests, the latter prioritizes those tests among the non-redundant ones that are more likely to detect an assertion violation. Therefore, our experiments show the best results for the combination of both.

\section{CONDITION INFERENCE}

Our may-unverified and must-unverified conditions reflect whether the premises of assertions further down in the control flow hold. In that sense, they resemble weakest preconditions [20]: a may-unverified condition is the negation of the weakest condition that implies that all premises further down hold; a must-unverified condition is the weakest condition that implies that all premises do not hold. Precisely computing such conditions, for instance via weakest precondition calculi [33], abstract interpretation [15], or predicate abstraction [30, 4], is too expensive for our purpose; the overhead of computing the conditions precisely would efface the benefits of pruning tests. Therefore, we use a rather coarse over-approximation of may- and must-unverified conditions that can be computed efficiently and is sufficiently precise to prune and prioritize tests effectively.

We first abstract the unit under test to a non-deterministic boolean program [5] where all variables are assumption identifiers. This step is an efficient syntactic program transformation. The abstraction is sound, that is, each execution of the concrete program is included in the set of executions of the abstract program. Therefore, a condition that guarantees that all premises hold (or are violated) in the abstract program provides the same guarantee for the concrete program. The may-unverified and must-unverified conditions can then be computed efficiently using abstract interpretation of the abstract program over a simple abstract domain.

\subsection{Abstraction}

We abstract a concrete program to a boolean program, where all boolean variables are assumption identifiers. In the abstract program, all expressions that do not include assumption identifiers are replaced by non-deterministically chosen values, which, in particular, replaces conditional control flow by non-determinism. Moreover, the abstraction removes assertions that have been fully verified, that is, where the premise is the literal true or includes true as a disjunct.

We present the abstraction for a simple concrete programming language with the following statements: assumed statements, assertions, method calls, conditionals, loops, and assignments. Besides conditional statements and loops with non-deterministic guards, the abstract language provides the following statements:

- initialization of assumption identifiers: var a $:=$ true,

- updates to assumption identifiers: $a:=a \& \& *$, where * denotes a non-deterministic (boolean) value,

- assertions: assert * verified $A$, where $A \not \equiv$ true, and

- method calls: call $M_{f}$, where $M_{f}$ is a fully-qualified method name and the receiver and arguments have been abstracted away.

Note that we desugar assumed statements into initializations and updates of assumption identifiers, which allows us to treat modular and whole-program analyses uniformly even though they require a different encoding of assumed statements (Sect. 2.1).

To abstract a program, we recursively apply the following transformations to its statements:

- an assumption assumed $P$ as a is rewritten to an assumption identifier initialization var a $:=$ true (at the appropriate program point, as discussed above) and an update a $:=$ a $\& \& *$,

- an assertion assert $P$ verified $A$ is transformed into assert * verified $A$, if $A$ is not trivially true, and omitted otherwise,

- a conditional statement if (b) $S_{0}$ else $S_{1}$ is rewritten to if $(*) S_{0}^{\prime}$ else $S_{1}^{\prime}$, where $S_{0}^{\prime}$ and $S_{1}^{\prime}$ are the results of recursively rewriting the statements $S_{0}$ and $S_{1}$,

- a loop while (b) $S$ is rewritten to while (*) $S^{\prime}$, where $S^{\prime}$ is the result of recursively rewriting statement $S$, 


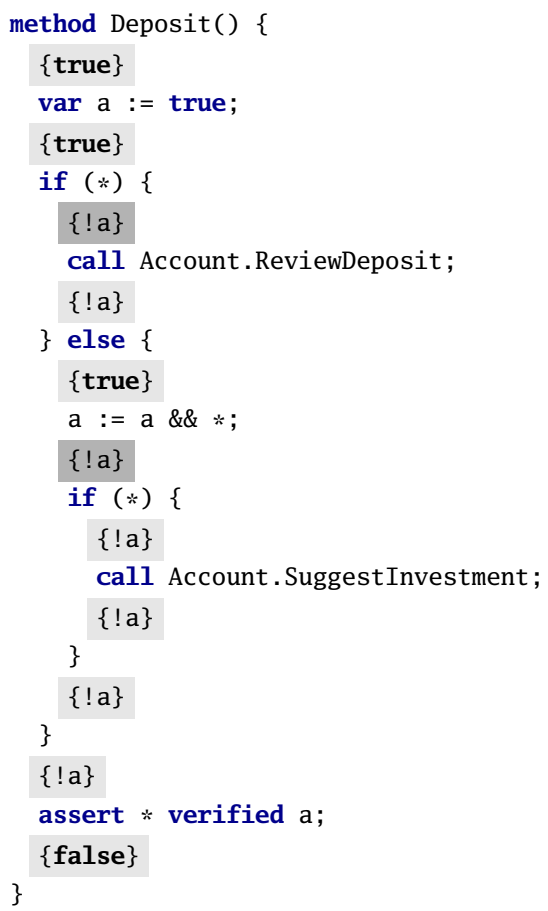

Figure 4: The abstraction of method Deposit from Fig. 2. The gray boxes (light and dark) show the inferred may-unverified conditions. The conditions that are used for the may-unverified instrumentation are shown in dark gray boxes.

- a method call r.M(..) is rewritten to call $M_{f}$, where $M_{f}$ is the fully-qualified name of $M$, and

- assignments are omitted.

Fig. 4 shows the abstraction of method Deposit from Fig. 2 . The gray boxes (light and dark) show the inferred mayunverified conditions, as we explain in the next subsection.

Soundness. The abstraction described above is sound, that is, each execution of the concrete program is included in the set of executions of the corresponding abstract program. The abstraction preserves the control structure of each method, but makes the control flow non-deterministic, which enlarges the set of possible executions. All other occurrences of expressions (in assumed statements, assertions, and calls) are replaced by non-deterministic values of the appropriate type, which also enlarges the set of possible executions. Once all occurrences of variables have been replaced by non-deterministic values, assignments do not affect program execution and can, thus, be omitted.

\subsection{May-unverified conditions}

A may-unverified condition expresses that some execution from the current program point onward may be unverified. We compute this condition for each program point in two steps. First, we compute the weakest condition at the corresponding program point in the abstract program that implies that all executions are verified. Since the set of executions of the abstract program subsumes the set of concrete executions, this condition also implies that all concrete executions are verified (although for the concrete execution, the computed condition is not necessarily the weakest such condition). Second, we negate the computed condition to obtain a may-unverified condition.
Inference. To compute the weakest condition that implies that all executions from a program point onward are verified, we define a predicate transformer $W P$ on abstract programs. If $W P(S, R)$ holds in a state, then the premise of each assertion in each execution of statement $S$ from that state holds and, if the execution terminates, $R$ holds in the final state. For a modular analysis such as Clousot, calls are encoded by asserting their precondition, reflecting their side effects, and assuming their postcondition; since our abstract programs omit all information about program variables, the latter two do not occur in the abstract program. Defining an inter-procedural $W P$ is of course also possible. Thus, we define $W P$ as follows:

- WP(assert * verified $A, R) \equiv A \wedge R$,

- WP(a := true, $R) \equiv R[$ a $:=$ true $]$, denoting the substitution of a by true in $R$, and

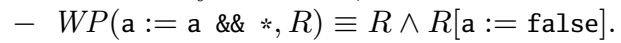

The semantics of sequential composition, conditionals, and loops is standard [20].

The may-unverified condition for a statement $S$ is the negation of the weakest precondition:

$$
\operatorname{MAY}(S) \equiv \neg W P(S, \text { true })
$$

In our implementation, we compute for each program point the may-unverified condition for the program fragment from this point onward. The computation is done using backward abstract interpretation over a set of cubes (that is, conjunctions of assumption identifiers or their negations). In the presence of loops, we use a fixed-point computation.

The (light and dark) gray boxes in Fig. 4 show the mayunverified conditions at each program point (assuming methods ReviewDeposit and SuggestInvestment have no preconditions). The may-unverified inference propagates meaningful information only up until the non-deterministic update is reached, which corresponds to the assumed statement. Specifically, on line 10, we infer true because the abstraction loses the information that would be needed to compute a stronger may-unverified condition. So, in return for an efficient condition inference, we miss some opportunities for aborting and pruning redundant tests.

Instrumentation. Since each execution of the concrete program corresponds to an execution of the abstract program, we can instrument the concrete program by adding an assume $C$ statement at each program point, where $C$ is the may-unverified condition at the corresponding program point in the abstract program. As we explained in Sect. 2.2, these statements abort redundant test cases and contribute constraints that guide DSE toward unverified executions.

To avoid redundant constraints that would slow down DSE, we omit assume statements when the may-unverified condition is trivially true or not different from the condition at the previous program point, as well as the assume false statement at the end of the unit under test. Therefore, out of all the conditions inferred for the example in Fig. 4, we use only the ones on lines 6 and 12 to instrument the program, which leads to the assumptions on lines 4 and 9 of Fig. 2 and guides DSE as described in Sect. 2.2.

\subsection{Must-unverified conditions}

A must-unverified condition expresses that (1) each execution from the program point onward contains at least one assertion and (2) on each execution, the premise of each assertion evaluates to false. 


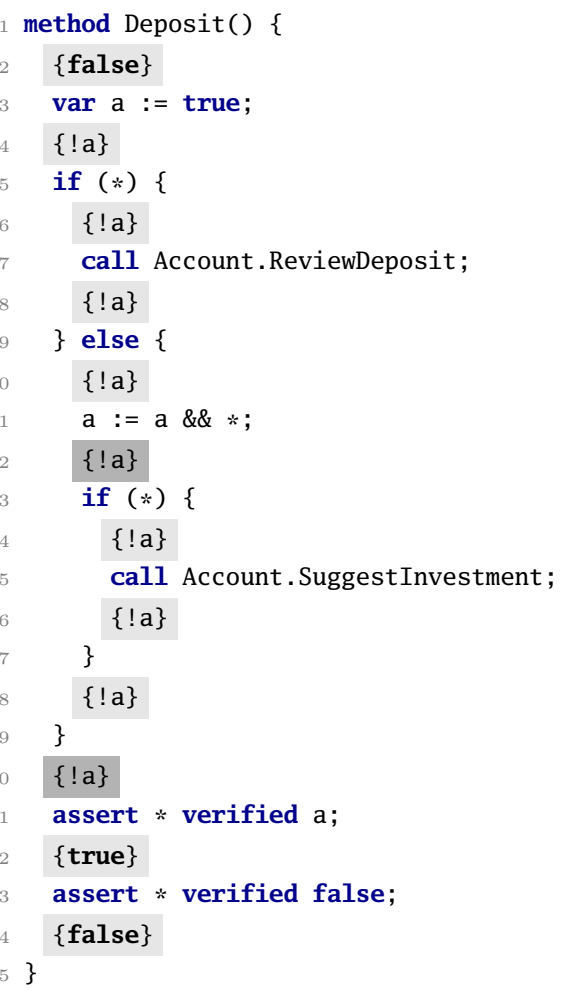

Figure 5: The abstraction of a variant of method Deposit from Fig. 2 that contains an additional unverified assertion at the end of the method (see Sect. 2.2). The gray boxes show the inferred mustunverified conditions. The conditions that are used for the must-unverified instrumentation are shown in dark gray boxes.

Inference. We infer the two properties that are entailed by a must-unverified condition separately via two predicate transformers MUST assert and $\operatorname{MUST}_{\text {all }}$. If $\operatorname{MUsT}_{\text {assert }}(S, R)$ holds in a state, then each execution of statement $S$ from that state encounters at least one assertion or terminates in a state in which $R$ holds. If $\operatorname{Must}_{\text {all }}(S, R)$ holds in a state, then the premise of each assertion in each execution of statement $S$ from that state does not hold and, if $S$ terminates, $R$ holds. Both transformers yield the weakest condition that has these properties. Consequently, we obtain the weakest must-unverified condition for an abstract statement $S$ as follows:

$$
\operatorname{Must}(S) \equiv \operatorname{Must}_{\text {assert }}(S, \text { false }) \wedge \operatorname{Must}_{\text {all }}(S, \text { true })
$$

MUST $_{\text {assert }}$ and MUST $_{\text {all }}$ are defined analogously to WP (see Sect. 3.2), except for the treatment of assertions:

$$
\begin{aligned}
& \operatorname{MUST}_{\text {assert }}(\text { assert * verified } A, R) \equiv t \text { true } \\
& \text { MUST all } \left._{\text {assert }} * \text { verified } A, R\right) \equiv \neg A \wedge R
\end{aligned}
$$

The definition for MUST assert expresses that, at a program point before an assertion, property (1) holds, that is, the remaining execution (from that point on) contains at least one assertion. The definition for Must all expresses that the premise $A$ must evaluate to false, and that $R$ must hold to ensure that the premises of subsequent assertions do not hold either.

Fig. 5 shows the abstraction of a variant of Deposit from Fig. 2 that contains an additional unverified assertion at the end of the method (see Sect. 2.2). The (light and dark) gray boxes show the inferred must-unverified conditions. Compared to the may-unverified conditions, the must-unverified conditions are stronger, that is, information is usually propagated further up in the control flow. Whereas the unverified assertion at the end of this example causes the mayunverified conditions to be trivially true, the must-unverified inference obtains conditions that can be used to prioritize test cases.

Instrumentation. To prioritize tests that satisfy their must-unverified conditions, we instrument the concrete program with tryfirst $C$ statements, where $C$ is the mustunverified condition at the corresponding program point in the abstract program. This statement causes DSE to prefer test inputs that satisfy condition $C$. More specifically, when a tryfirst $C$ statement is executed for the first time, it adds $C$ to the path condition to force DSE to generate inputs that satisfy condition $C$. Note however, that unlike the constraints added by assume statements, this constraint may be dropped by the DSE to also explore executions where the condition is violated. If during this first execution of the statement condition $C$ is violated, then the test case is interrupted and will be re-generated later when condition $C$ can no longer be satisfied. So the tryfirst statement influences the order in which test cases are generated, but never aborts or prunes tests. Nevertheless, the order is important because DSE is typically applied until certain limits (for instance, on the overall testing time or the number of test cases) are reached. Therefore, exploring non-redundant test cases early increases effectiveness.

Pex supports primitives for expressing tryfirst $C$ statements easily, as instrumentation. Alternatively, other tools may encode them by placing additional branches into the code and customizing the search strategy to prefer the branch where $C$ holds.

To avoid wasting time on interrupting tests that will be re-generated later, our implementation enforces an upper bound on the number of interrupts that are allowed per unit under test. When this upper bound is exceeded, all remaining tryfirst statements have no effect.

As illustrated by lines 4, 6, 8, and 10 in Fig. 5, the mustunverified condition at some program points evaluates to false for all executions. Instrumenting these program points would lead to useless interruption and re-generation of test cases. To detect such cases, we apply constant propagation and do not instrument program points for which the mustunverified conditions are trivially true or false. Moreover, we omit the instrumentation for conditions that are not different from the condition at the previous program point. Therefore, out of all the conditions inferred for the example in Fig. 5, we use only the ones on lines 12 and 20 to instrument the program, which prioritize test cases that lead to an arithmetic overflow on line 10, as discussed in Sect. 2.2.

\subsection{Combined instrumentation}

As we explained in Sect. 2.2, the may-unverified instrumentation aborts and prunes redundant tests, while the must-unverified instrumentation prioritizes test cases that are more likely to detect an assertion violation. One can, therefore, combine both instrumentations such that DSE (1) attempts to first explore program executions that must be unverified, and (2) falls back on executions that may be unverified when the former is no longer feasible. 
The combined instrumentation includes both the assume statements from the may-unverified instrumentation and the tryfirst statements from the must-unverified instrumentation. The tryfirst statement comes first. Whenever we can determine that the must-unverified and may-unverified conditions at a particular program point are equivalent, we omit the tryfirst statement, because any interrupted and re-generated test case would be aborted by the subsequent assume statement anyway.

\section{EXPERIMENTS}

In this section, we give an overview of our implementation and present our experimental results. They show that, compared to dynamic symbolic execution alone, our technique produces smaller test suites, covers more unverified executions, and reduces testing time. They also show that the combined instrumentation is more effective than the mayunverified or the must-unverified instrumentation alone.

\subsection{Implementation}

We have implemented our technique for the .NET static analyzer Clousot [23] and the DSE tool Pex [40]. Our tool chain consists of four subsequent stages: (1) static analysis and verification-annotation instrumentation, (2) mayunverified and must-unverified instrumentation, (3) runtime checking, and (4) dynamic symbolic execution.

The first stage runs Clousot on a given .NET program, which contains code and optionally specifications expressed in Code Contracts [22], and instruments the sources of unsoundness and partial verification results of the analyzer using our verification annotations. For this purpose, we have implemented a wrapper around Clousot that uses the debug output emitted during the static analysis to instrument the program (at the binary level). Note that Clousot performs a modular analysis, and thus, the verification annotations are local to the containing methods.

We have elicited a complete list of Clousot's unsound assumptions by studying publications, extensively testing the tool, and having numerous discussions with its designers. We encoded most of these assumptions with our verification annotations [13].

The second stage of the tool chain adds the may-unverified, must-unverified instrumentation, or their combination to the annotated program.

In the third stage, we run the existing Code Contracts binary rewriter to transform any Code Contracts specifications into runtime checks. We then run a second rewriter that transforms all the assumed statements and assertions of the annotated program into assignments and assumptions, as described in Sect. 2.1.

In the final stage, we run Pex on the instrumented code.

\subsection{Experimental evaluation}

In the rest of this section, we describe the setup for the evaluation of our technique and present experiments that evaluate its benefits.

Setup. For our experiments, we used 101 methods (written in $\mathrm{C \# )}$ from nine open-source projects and from solutions to 13 programming tasks on the Rosetta Code repository. A complete list of the methods used in our evaluation can be found in Christakis' Ph.D. thesis [11]. We selected only methods for which Pex can automatically (that is, without user-provided factories) produce at least one test case that passes the method's parameter validation (between 1 and 25 methods per project or task).

In Clousot, we enabled all checks, set the warning level to the maximum, and disabled all inference options. In Pex, we set the maximum number of branches, conditions, and execution tree nodes to 100,000 , and the maximum number of concrete runs to 30 . Without any instrumentation, 61 methods reach this maximum number of runs, and 35 are tested exhaustively, which gives an indication of the complexity of the selected methods.

In our experiments, we allowed up to 4 test interrupts per method under test when these are caused by tryfirst statements (see Sect. 3.3). We experimented with different such bounds (1, 2, 4, and 8) on 25 methods from the suite of 101 methods. For an upper bound of 4 for the number of allowed interrupts per method, DSE strikes a good balance between testing time and the number of detected bugs.

We used a machine with a quad-core CPU (Intel Core i7$4770,3.4 \mathrm{GHz}$ ) and $16 \mathrm{~GB}$ of RAM for these experiments.

Performance of static analysis and instrumentation. On average, Clousot analyzes each method from our suite in 1.9 seconds. The may-unverified and must-unverified instrumentations are very efficient. On average, they need 22 milliseconds per method when combined.

Configurations. To evaluate our technique, we use the following configurations:

- UV: unverified code.

Stages 1 and 2 of the tool chain are not run.

- PV: partially-verified code.

Stage 2 of the tool chain is not run.

- MAY: partially-verified code, instrumented with mayunverified conditions.

All stages of the tool chain are run. Stage 2 adds only the may-unverified instrumentation.

- MUST: partially-verified code, instrumented with mustunverified conditions.

All stages of the tool chain are run. Stage 2 adds only the must-unverified instrumentation.

- MAY $\times M U S T$ : partially-verified code, instrumented with may-unverified and must-unverified conditions.

All stages of the tool chain are run. Stage 2 adds the combined may- and must-unverified instrumentation.

Fig. 6 shows the number of tests that each configuration generated for the 101 methods, categorized as nonredundant and failing, as non-redundant and successful, or as redundant tests. A failing test is a test that terminates abnormally, whereas a successful one terminates normally. Tests that terminate on exceptions that are explicitly thrown by the method under test, for instance, for parameter validation, are considered successful. To determine the redundant tests, we counted the tests in which the premises of all encountered assertions hold. Note that the figure does not include tests that are interrupted when a condition in a tryfirst statement is violated (since these tests are regenerated-and counted-later).

The results of DSE alone, that is, of $U V$, do not significantly differ from those of $P V$ in terms of the total number of tests and the number of non-redundant tests generated. This confirms that the instrumentation from stage 1 alone, without the may-unverified and must-unverified instrumentation, does not reduce the test effort significantly 


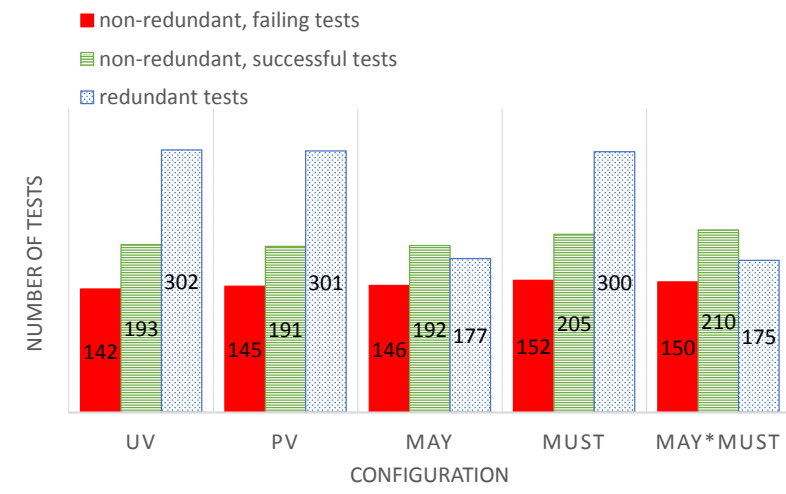

Figure 6: The tests generated by each configuration, categorized as non-redundant and failing, as non-redundant and successful, or as redundant tests. $M A Y \times M U S T$ generates $\mathbf{1 6 . 1 \%}$ fewer tests, but $\mathbf{7 . 1 \%}$ more non-redundant tests than $P V$, including 5 additional failing tests.

for partially-verified methods, as we explained in Sect. 2.2. Note that this result does not contradict the results of our previous work [12]. First, that work used a different static analyzer whose (artificial) sources of unsoundness affected fewer methods than Clousot's, leading to a much larger portion of fully-verified methods. Second, the improvements observed in our earlier work were mostly caused by exempting fully-verified methods completely from the test stage, whereas here, we apply stage 4 to all methods and rely on our may-instrumentation to prune the entire search space.

For the following experiments, we use configuration $P V$ as the baseline to highlight the benefits of the may-unverified and must-unverified inference over our earlier work [12].

Smaller test suites. The may-unverified instrumentation causes DSE to abort tests leading to verified executions and to prune verified parts of the search space. As a result, DSE generates smaller test suites. Fig. 6 shows that, in total, MAY generates $19.2 \%$ fewer tests and $M A Y \times M U S T$ generates $16.1 \%$ fewer tests than $P V$. The differences in the total number of tests for configurations without the mayunverified instrumentation are minor.

Fig. 7 compares the total number of generated tests (including aborted tests) by $P V$ and $M A Y$ per method. For many methods, $M A Y$ produces fewer tests, as shown by the negative values. However, for some methods, $M A Y$ generates more tests than $P V$. This happens when pruning verified parts of the search space guides DSE toward executions that are easier to cover within the exploration bounds of Pex (for instance, maximum number of branches).

More unverified executions. Although configurations $M A Y$ and $M A Y \times M U S T$ generate smaller test suites in comparison to $P V$, they do not generate fewer non-redundant tests, as shown in Fig. 6. In other words, they generate at least as many non-redundant tests as $P V$, thus covering at least as many unverified executions.

The must-unverified instrumentation prioritizes test inputs that lead to more premise violations. In comparison to the may-unverified conditions, the must-unverified conditions are stronger and their instrumentation is usually added further up in the control flow. As a result, MUST and $M A Y \times M U S T$ guide DSE to cover unverified executions ear-

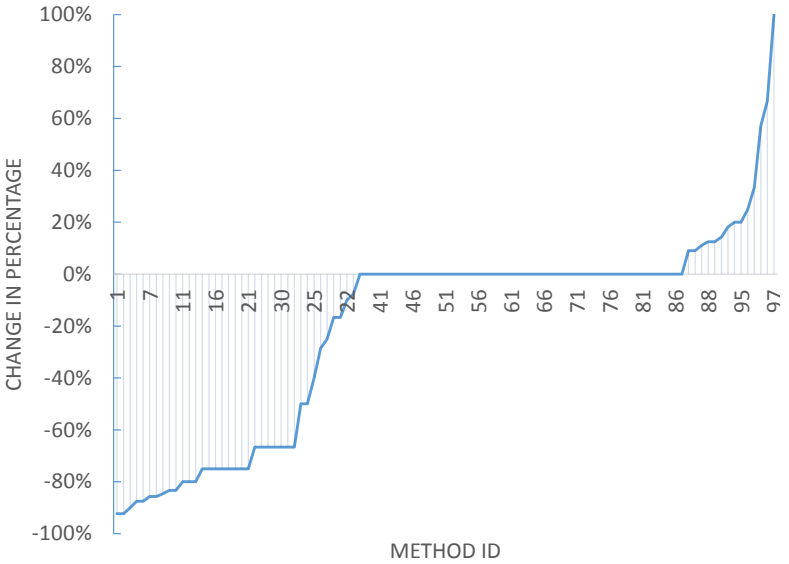

Figure 7: Change in total number of tests generated for each of the 101 methods by configuration $M A Y$ in comparison to $P V$ (in percentage). Negative values indicate that $M A Y$ produces fewer tests.

lier and may allow it to generate more non-redundant tests within the exploration bounds. As shown in Fig. 6, configuration $M U S T$ generates $6.3 \%$ more non-redundant tests than $P V$ and $5.6 \%$ more than $M A Y(M A Y \times M U S T$ produces $7.1 \%$ resp. $6.5 \%$ more non-redundant tests). By generating more such tests, we increase the chances of producing more failing tests. In fact, $M U S T$ generates $4.8 \%$ more failing tests than $P V$ and $4.1 \%$ more than $M A Y(M A Y \times M U S T$ produces $3.4 \%$ resp. $2.7 \%$ more failing tests).

MUST typically generates more non-redundant tests for methods in which Clousot detects errors, that is, for methods with unverified assertions. In such methods, the mayunverified instrumentation is added only after the unverified assertions in the control flow (if the conditions are nontrivial), thus failing to guide DSE toward unverified executions early on, as discussed in Sect. 2.2.

Shorter testing time. To compare the testing time of the different configurations, we considered only methods for which all configurations generated the same number of nonredundant tests. This is to ensure a fair comparison; for these methods, all configurations achieved the same coverage of unverified executions. This experiment involved 72 out of the 101 methods, and the time it took for each configuration to test these methods is shown in Fig. 8. As expected, pruning verified parts of the search space with the may-unverified instrumentation is very effective. In particular, configuration $M A Y$ is $51.7 \%$ faster and configuration $M A Y \times M U S T$ is $52.4 \%$ faster than $P V$. The difference between $P V$ and MUST is caused by a few outliers, for which $P V$ runs more than twice as long. The MUST instrumentation affects the order in which execution paths are explored. Even though the same number of non-redundant tests is generated, the generated tests could exercise different paths, leading to different constraint solving times.

Note that Fig. 8 does not include the time of the static analysis for two reasons. First, Clousot is just one way of obtaining verification results. Second, the goal of our work is to efficiently complement existing verification results with test case generation; we assume that the static analysis is run anyway to achieve a more thorough scrutiny of the code. Recall that the overhead of the instrumentation is negligible.

Even though $M A Y$ is overall much faster than $P V$, there 


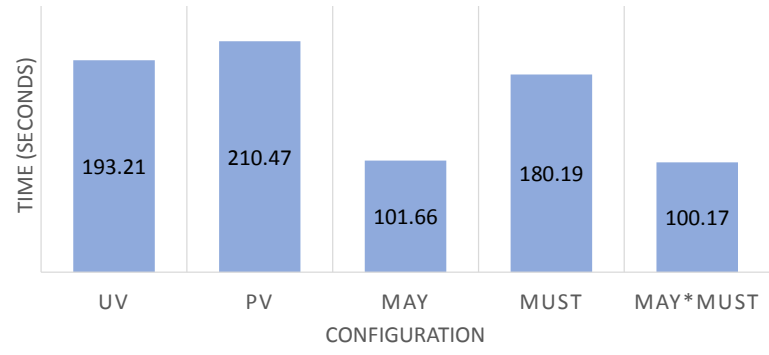

Figure 8: Testing time for each configuration. We only considered methods for which all configurations generated the same number of non-redundant tests. $M A Y \times M U S T$ is $\mathbf{5 2 . 4 \%}$ faster than $P V$.

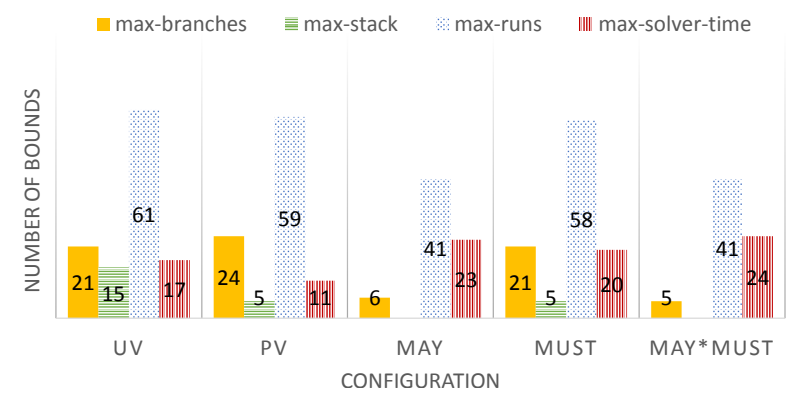

Figure 9: The exploration bounds reached by each configuration. $M A Y$ and $M A Y \times M U S T$ overall reach fewer bounds than $P V$.

were methods for which the testing time for $M A Y$ was longer in comparison to $P V$. This is the case when constraint solving becomes more difficult due to the inferred conditions. In particular, it might take longer for the constraint solver to prove that an inferred condition at a certain program point is infeasible.

Fewer exploration bounds reached. During its exploration, DSE may reach bounds that prevent it from covering certain, possibly failing, execution paths. There are four kinds of bounds that were reached during our experiments: - max-branches: maximum number of branches that may be taken along a single execution path;

- max-stack: maximum number of active call frames on the stack at any time during a single execution path;

- max-runs: maximum number of runs that will be tried during an exploration (each run uses different inputs but some runs are not added to the test suite if they do not increase coverage);

- max-solver-time: maximum time that the constraint solver has to find inputs that will cause an execution path to be taken.

Fig. 9 shows the exploration bounds in Pex that were reached by each configuration when testing all 101 methods. MAY, MUST, and MAY $\times M U S T$ reach the maxsolver-time bound more often than $P V$. This is because our instrumentation introduces additional conjuncts in the path conditions, occasionally making constraint solving harder. Nevertheless, configurations $M A Y$ and $M A Y \times M U S T$ overall reach significantly fewer bounds than $P V$ (for instance, the max-stack bound is never reached) by pruning verified parts of the search space. This helps in alleviating an inherent limitation of symbolic execution by building on results from tools that do not suffer from the same limitation.
Winner configuration. Configuration $M A Y \times M U S T$ generates the second smallest test suite containing the largest number of non-redundant tests and the smallest number of redundant tests (Fig. 6). This is achieved in the shortest amount of time for methods with the same coverage of unverified executions across all configurations (Fig. 8) and by reaching the smallest number of exploration bounds (Fig. 9).

Therefore, $M A Y \times M U S T$ effectively combines the benefits of both the may-unverified and must-unverified instrumentation to prune parts of the search space that lead only to verified executions as well as to identify and prefer test inputs that lead to unverified executions as soon as possible.

Note that, in practice, these benefits should be independent of the exploration strategy in the underlying DSE. For methods whose exploration does not reach any bounds, the order in which the tests are generated is obviously not relevant. For the remaining methods, we do not expect an exploration strategy to significantly affect how often our instrumentation is hit because Clousot makes unsound assumptions for various expressions and statements and, thus, assumed statements are spread across the method body. We have confirmed this expectation by running the $M A Y \times$ MUST configuration with different exploration strategies on 20 methods for which exploration bounds were reached. The differences between all strategies (breadth-first, random search, and Pex's default search strategy) were negligible.

Threats to validity. We identified the following threats to the validity of our experiments:

- Sample size: We used 101 methods from nine C\# projects and from solutions to 13 programming tasks.

- Static analyzer: For our experiments, we used a modular (as opposed to whole-program) static analyzer, namely, Clousot. Moreover, our experimental results depend on the deliberate sources of unsoundness and verification results of this particular analyzer. Note that there are a few sources of unsoundness in Clousot that our tool chain does not capture [13], for instance, about reflection or unmanaged code.

- Soundly-analyzed methods: 23 out of the 101 methods contain no assumed statements. Clousot reports no warning for 16 of them, and thus, these methods are fully verified and our may-unverified instrumentation prunes the entire search space. However, our results are not significantly affected by including these methods: the difference in running times with and without fully-verified methods is minor (e.g., $M A Y \times M U S T$ is still $50.3 \%$ faster than $P V)$.

- Failing tests: The failing tests generated by each configuration do not necessarily reveal bugs in the containing methods. This is inherent to unit testing since methods are tested in isolation rather than in the context of the entire program. However, 50 out of the 101 methods validate their parameters (and for 10 methods no parameter validation was necessary), which suggests that programmers did intend to prevent failures in these methods.

\section{RELATED WORK}

Many static analyzers for mainstream languages such as HAVOC [3], Spec\# [6], and ESC/Java [24] deliberately make unsound assumptions to increase automation, improve performance, and reduce the number of false positives and the annotation overhead for the programmer [36]. Our tech- 
nique can effectively complement these analyzers by DSE.

Integration of static analysis and testing. Various approaches combine static analysis and automatic test case generation to determine whether an error reported by the static analysis is spurious and to reduce the search space for the test case generator. For example, Check 'n' Crash [17] is an automated defect detection tool that integrates the unsound ESC/Java static checker with the JCrasher [16] test case generator. Check 'n' Crash was later integrated with Daikon [21] in the DSD-Crasher tool [18]. Similarly, DyTa [25] integrates Clousot with Pex. Like our work, all of these approaches use results from the static analysis to guide test case generation toward the errors reported by the static analysis and to prune parts of the search space during testing. However, in contrast to our work, they ignore the unsoundness of the static analysis: each assertion for which the static analysis does not report an error is considered soundly verified, even if the analysis makes unsound assumptions. Consequently, these approaches may prune unverified executions, whereas our technique retains all executions that are not fully verified and, therefore, may reveal errors missed by the unsound static analysis.

SANTE [10] uses a sound value analysis (in combination with program slicing) to prune those execution paths that do not lead to unverified assertions. In contrast, our work supports the common case that a static analysis is unsound.

Several analyses combine over- and under-approximations of the set of program executions. Counterexample-guided abstraction refinement (CEGAR) [14] exploits the abstract counterexample trace of a failing proof attempt to suggest a concrete trace that might reveal a real error. If, however, the abstract trace refers to a spurious error, the abstraction is refined in such a way that subsequent verification attempts will not reproduce the infeasible abstract trace. YOGI [29, 37] switches between static analysis and DSE both to prove properties and find bugs, without reporting false positives. Specifically, YOGI uses two different abstract domains, one (not-)may abstraction for proving a property and one must abstraction for disproving a property. The two abstractions are used simultaneously, communicate with each other, and refine each other for either finding a proof or a bug. To obtain an over-approximation of the set of program executions, these approaches rely on a sound analysis. In contrast, our work supports the common case that a static analysis is unsound, that is, neither over- nor under-approximates the set of program executions (the analysis may have both false positives and false negatives). Soundly-verified executions and executions for which the analysis reports an error are handled similarly to work based on over-approximations: we prune soundly-verified executions during test case generation, and use an under-approximation (testing) to find bugs and identify spurious errors among the executions for which the analysis reports an error. The novelty of our work is that we also handle executions that are verified unsoundly, that is, under unsound assumptions. Our annotations make these assumptions explicit (in other words, they express which executions one would have to add to the set of analyzed executions for it to become a sound over-approximation). These executions are then targeted by an under-approximation.

A recent approach [19] starts by running a conditional model checker [7] on a program, and then tests those parts of the state space that were not covered by the model checker (for instance, due to timeouts). More specifically, the model checker produces an output condition, which captures the safe states and is used to produce a residual program that can be subsequently tested. Unlike an instrumented program in our technique, the residual program can be structurally very different from the original program. As a result, its construction can take a significant amount of time, as the authors point out. Furthermore, this approach can characterize assertions only as either fully verified or unverified on a given execution path. It is not clear how to apply this approach in a setting with static analysis tools that are not fully sound $[36,13]$ without reducing its effectiveness.

Dynamic symbolic execution. Testing and symbolically executing all feasible program paths is not possible in practice. The number of feasible paths can be exponential in the program size, or even infinite in the presence of inputdependent loops.

Existing DSE tools alleviate path explosion using search strategies and heuristics that guide the search toward interesting paths while pruning the search space. These strategies typically optimize properties such as "deeper paths" (in depth-first search), "less-traveled paths" [35], "number of new instructions covered" (in breadth-first search), or "paths specified by the programmer" [39]. For instance, SAGE [28] uses a generational-search strategy in combination with simple heuristics, such as flip count limits and constraint subsumption. Other industrial-strength tools, like Pex, also use similar techniques. As we explained in Sect. 4.2, the benefits of our approach are independent of the exploration strategy in the underlying DSE. Our technique resembles a search strategy in that it optimizes unverified executions, prunes verified executions, and is guided by verification annotations, instead of properties like the above.

Compositional symbolic execution $[26,1]$ has been shown to alleviate path explosion. Dynamic state merging [32] and veritesting [2] achieve this by merging sub-program searches, while RWset [8] prunes searches by dynamically computing variable liveness. By guiding DSE toward unverified program executions, our technique also alleviates path explosion. In particular, the may-unverified instrumentation causes DSE to abort tests that lead to verified executions and to prune parts of the search space. Moreover, since our technique does not require a particular DSE algorithm, it can be combined with any of the above approaches by running them on a program that contains our instrumentation.

\section{CONCLUSION}

We have presented a technique for complementing partial verification results by automatic test case generation. Our technique causes dynamic symbolic execution to abort tests that lead to verified executions, consequently pruning parts of the search space, and to prioritize tests that are more likely to detect an assertion violation. It is applicable to any program with verification annotations, either inserted automatically by a (possibly unsound) static analysis or manually, for instance, during a code review. Our work suggests a novel way to combine static analysis and testing in order to maximize software quality, and investigates to what extent unsound static analysis reduces the test effort.

Acknowledgments. We thank Cristian Cadar, Patrice Godefroid, Thomas Gross, and Nikolai Tillmann for their valuable help and feedback. We are also grateful to the anonymous reviewers for their constructive comments. 


\section{REFERENCES}

[1] S. Anand, P. Godefroid, and N. Tillmann. Demand-driven compositional symbolic execution. In TACAS, volume 4963 of $L N C S$, pages 367-381. Springer, 2008.

[2] T. Avgerinos, A. Rebert, S. K. Cha, and D. Brumley. Enhancing symbolic execution with veritesting. In ICSE, pages 1083-1094. ACM, 2014.

[3] T. Ball, B. Hackett, S. K. Lahiri, S. Qadeer, and J. Vanegue. Towards scalable modular checking of user-defined properties. In VSTTE, volume 6217 of LNCS, pages 1-24. Springer, 2010.

[4] T. Ball, R. Majumdar, T. D. Millstein, and S. K. Rajamani. Automatic predicate abstraction of $\mathrm{C}$ programs. In PLDI, pages 203-213. ACM, 2001.

[5] T. Ball and S. K. Rajamani. Boolean programs: A model and process for software analysis. Technical Report MSR-TR-2000-14, Microsoft Research, 2000.

[6] M. Barnett, M. Fähndrich, K. R. M. Leino, P. Müller, W. Schulte, and H. Venter. Specification and verification: The Spec\# experience. CACM, 54:81-91, 2011.

[7] D. Beyer, T. A. Henzinger, M. E. Keremoglu, and P. Wendler. Conditional model checking: A technique to pass information between verifiers. In FSE, pages 57-67. ACM, 2012.

[8] P. Boonstoppel, C. Cadar, and D. R. Engler. RWset: Attacking path explosion in constraint-based test generation. In $T A C A S$, volume 4963 of $L N C S$, pages 351-366. Springer, 2008.

[9] C. Cadar and D. R. Engler. Execution generated test cases: How to make systems code crash itself. In SPIN, volume 3639 of $L N C S$, pages 2-23. Springer, 2005.

[10] O. Chebaro, N. Kosmatov, A. Giorgetti, and J. Julliand. The SANTE tool: Value analysis, program slicing and test generation for $\mathrm{C}$ program debugging. In $T A P$, volume 6706 of $L N C S$, pages 78-83. Springer, 2011.

[11] M. Christakis. Narrowing the Gap between Verification and Systematic Testing. PhD thesis, ETH Zurich, 2015.

[12] M. Christakis, P. Müller, and V. Wüstholz. Collaborative verification and testing with explicit assumptions. In FM, volume 7436 of $L N C S$, pages 132-146. Springer, 2012.

[13] M. Christakis, P. Müller, and V. Wüstholz. An experimental evaluation of deliberate unsoundness in a static program analyzer. In $V M C A I$, volume 8931 of LNCS, pages 336-354. Springer, 2015.

[14] E. M. Clarke, O. Grumberg, S. Jha, Y. Lu, and H. Veith. Counterexample-guided abstraction refinement. In $C A V$, volume 1855 of $L N C S$, pages 154-169. Springer, 2000.

[15] P. Cousot and R. Cousot. Abstract interpretation: A unified lattice model for static analysis of programs by construction or approximation of fixpoints. In $P O P L$, pages 238-252. ACM, 1977.

[16] C. Csallner and Y. Smaragdakis. JCrasher: An automatic robustness tester for Java. SPE, 34:1025-1050, 2004.

[17] C. Csallner and Y. Smaragdakis. Check 'n' Crash:
Combining static checking and testing. In ICSE, pages 422-431. ACM, 2005.

[18] C. Csallner, Y. Smaragdakis, and T. Xie. DSD-Crasher: A hybrid analysis tool for bug finding. TOSEM, 17:1-37, 2008.

[19] M. Czech, M.-C. Jakobs, and H. Wehrheim. Just test what you cannot verify! In FASE, volume 9033 of LNCS, pages 100-114. Springer, 2015.

[20] E. W. Dijkstra. Guarded commands, nondeterminacy and formal derivation of programs. $C A C M$, 18:453-457, 1975.

[21] M. D. Ernst, J. H. Perkins, P. J. Guo, S. McCamant, C. Pacheco, M. S. Tschantz, and C. Xiao. The Daikon system for dynamic detection of likely invariants. Sci. Comput. Program., 69:35-45, 2007.

[22] M. Fähndrich, M. Barnett, and F. Logozzo. Embedded contract languages. In $S A C$, pages 2103-2110. ACM, 2010.

[23] M. Fähndrich and F. Logozzo. Static contract checking with abstract interpretation. In FoVeOOS, volume 6528 of $L N C S$, pages 10-30. Springer, 2010.

[24] C. Flanagan, K. R. M. Leino, M. Lillibridge, G. Nelson, J. B. Saxe, and R. Stata. Extended static checking for Java. In PLDI, pages 234-245. ACM, 2002.

[25] X. Ge, K. Taneja, T. Xie, and N. Tillmann. DyTa: Dynamic symbolic execution guided with static verification results. In ICSE, pages 992-994. ACM, 2011.

[26] P. Godefroid. Compositional dynamic test generation. In POPL, pages 47-54. ACM, 2007.

[27] P. Godefroid, N. Klarlund, and K. Sen. DART: Directed automated random testing. In $P L D I$, pages 213-223. ACM, 2005.

[28] P. Godefroid, M. Y. Levin, and D. A. Molnar. Automated whitebox fuzz testing. In $N D S S$, pages 151-166. The Internet Society, 2008.

[29] P. Godefroid, A. V. Nori, S. K. Rajamani, and S. Tetali. Compositional may-must program analysis: Unleashing the power of alternation. In $P O P L$, pages 43-56. ACM, 2010.

[30] S. Graf and H. Saïdi. Construction of abstract state graphs with PVS. In $C A V$, volume 1254 of $L N C S$, pages 72-83. Springer, 1997.

[31] G. J. Holzmann. Mars code. CACM, 57:64-73, 2014.

[32] V. Kuznetsov, J. Kinder, S. Bucur, and G. Candea. Efficient state merging in symbolic execution. In PLDI, pages 193-204. ACM, 2012.

[33] K. R. M. Leino. Efficient weakest preconditions. IPL, 93:281-288, 2005.

[34] K. R. M. Leino. Dafny: An automatic program verifier for functional correctness. In $L P A R$, volume 6355 of LNCS, pages 348-370. Springer, 2010.

[35] Y. Li, Z. Su, L. Wang, and X. Li. Steering symbolic execution to less traveled paths. In OOPSLA, pages 19-32. ACM, 2013.

[36] B. Livshits, M. Sridharan, Y. Smaragdakis, O. Lhoták, J. N. Amaral, B.-Y. E. Chang, S. Z. Guyer, U. P. Khedker, A. Møller, and D. Vardoulakis. In defense of soundiness: A manifesto. CACM, 58:44-46, 2015.

[37] A. V. Nori, S. K. Rajamani, S. Tetali, and A. V. 
Thakur. The YOGI project: Software property checking via static analysis and testing. In TACAS, volume 5505 of $L N C S$, pages 178-181. Springer, 2009.

[38] K. Sen, D. Marinov, and G. Agha. CUTE: A concolic unit testing engine for C. In $E S E C$, pages 263-272. ACM, 2005.

[39] K. Sen, H. Tanno, X. Zhang, and T. Hoshino. GuideSE: Annotations for guiding concolic testing. In $A S T$, pages 23-27. IEEE Computer Society, 2015.

[40] N. Tillmann and J. de Halleux. Pex-White box test generation for .NET. In TAP, volume 4966 of $L N C S$, pages 134-153. Springer, 2008.

[41] V. Wüstholz. Partial Verification Results. PhD thesis, ETH Zurich, 2015. 